\title{
Assessment of Cardiovascular Risk in Patients with Rheumatoid Arthritis
}
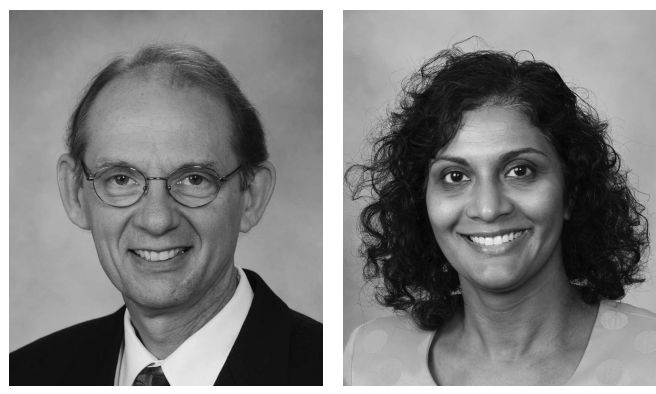

Patients with rheumatoid arthritis (RA) are at increased risk for cardiovascular disease (CVD), including peripheral vascular disease, stroke, myocardial infarction, and heart failure compared to the general population ${ }^{1,2}$. The absolute $\mathrm{CV}$ risk is estimated to be up to 2 -fold increased ${ }^{1,2}$. The risk is increased because of chronic, systemic inflammation, as well as premature atherosclerosis. In addition to the risk inherent to the underlying disease, conventional risk factors are major contributors to the increase in recurrence of CVD, including dyslipidemia, hypertension (HTN), obesity, smoking, and diabetes ${ }^{3}$.

In this edition of The Journal, Barber, et al report on the experience of using $\mathrm{CV}$ risk indicators in clinical practice ${ }^{4}$. In 2 cohorts of patients, 1 in which patients enrolled into an early RA disease clinic and the other where patients enrolled into a biologic registry, the authors report on the frequencies with which CVD indicators are assessed. These are summarized in a baseline 10-year Framingham Risk Score for those patients whose risk is identified by the Framingham Risk Score, age 30-74 years, and not taking a baseline statin. As anticipated, patients in the biologics cohort generally had more active disease, had worse functional status, were more likely to have erosive disease, and had extraarticular disease.

The quality indicators assessed as CVD risk factors in the study included evidence of discussion of increased CV risk with the patient, formal CV risk assessment, assessment of smoking status and offer of smoking cessation counseling, screening for HTN and communication of HTN findings to the patient's primary care provider, measurement of lipid profile, screening for diabetes, exercise counseling, and body mass index screening and lifestyle counseling, as well as documented attempts to reduce glucocorticoid usage and communication of the risk/benefits of antiinflammatory agents and cyclooxygenase- 2 inhibitors in terms of their risk for CVD occurrence ${ }^{4}$.

Although conducted in a subspecialty setting with high degree of awareness of $\mathrm{CV}$ risk, performance indicators of
$\mathrm{CV}$ risk assessment were variably obtained. High performance was obtained for baseline documentation of smoking, in $98 \%$ of patients, although only $17 \%$ of patients received smoking cessation counseling. Blood pressure was obtained at least $80 \%$ of clinic visits; however, communication to the primary care provider of HTN detection was rarely provided. Rates of screening for hyperlipidemia and diabetes were also high, performed in two-thirds of patients, whereas a yearly discussion of physical activity recommendations was pursued in only about one-third of patients in the first year of enrollment; and by year 2 , in only $15 \%$ of patients. On the other hand, there was a high degree of awareness of the importance of reducing glucocorticoid dose, with attempts to reduce dosing documented at virtually all visits, while communication regarding reduction of nonsteroidal antiinflammatory drug use, especially in those patients with medium or high risk of CVD, was low.

The results of this study demonstrate the practical difficulties of CVD assessment. The initial and longterm management recommendations that need to be pursued in the rheumatologic practice and in the primary care practice in these patients who are at higher risk for CVD have not been developed. Formal Framingham risk assessment, which is recognized to be inadequate and to underestimate overall the CV risk in patients with RA and other systemic inflammatory rheumatic diseases, was not commonly performed; and recommendations for $\mathrm{CV}$ risk reduction were variably pursued. The reasons are complex and likely relate not to lack of awareness of CVD disease risk but rather to the time-consuming nature of such risk assessment and communication of management recommendations to patients and their primary care physicians, even in a well-managed rheumatology practice setting. It is a fair statement that, at present, awareness of risk is not effectively translated into meaningful management recommendations.

Given the frequency of RA, the overall population disease burden of CVD in these patients is substantial. RA affects

See Cardiovascular care in RA, page 1965

Personal non-commercial use only. The Journal of Rheumatology Copyright (c) 2016 . All rights reserved. 
between 0.5 and 1.0 percent of the population in Northern Europe and North America ${ }^{5,6}$. Patients with RA have a worse mortality experience than patients who do not have RA, and CVD is a significant contributor to the mortality burden ${ }^{7}$. Indeed, of all the comorbidities affecting patients with RA, other than lung disease, CVD confers the greatest risk of premature mortality $8,9,10,11,12$. CVD accounts for about $40 \%$ to $50 \%$ of all deaths in $\mathrm{RA}^{1,2,10}$ and occurs a decade earlier than in a non-RA cohort ${ }^{10}$. In addition to the more traditional variables of $\mathrm{CV}$ risk, specific forms of heart disease also affect the morbidity and mortality of patients with RA, including pericardial disease, myocardial inflammation, cardiac amyloidosis, and nonatherosclerotic coronary disease, which may manifest as arrhythmias, particularly atrial fibrillation, ventricular arrhythmias, and valvular heart disease including valvular fibrosis, calcifications, rheumatoid nodules, and noninfectious endocarditis, especially in patients with high levels of joint disease activity and severity.

The central issue raised by Barber, et al is how to effectively screen for and diagnose CVD in RA. As suggested by the often lacking communication of risk assessment between rheumatologists and primary care providers documented in their study, there is confusion about which healthcare providers are responsible for recording and evaluating $\mathrm{CV}$ risk factors. This makes the implementation of recommendations a challenge with respect to modifying and following up on recommendations for lifestyle-related risk factors.

The nature of the healthcare system in different regions and countries is an additional factor affecting how recommendations for CVD risk modification are implemented in various healthcare settings. For example, in systems that are strongly primary care-oriented and in which specialists provide detailed management for rheumatic disease entities only, the general practitioner or internist is likely to assume the primary responsibility for these evaluations and management. In other systems, specialists including cardiologists may have primary responsibility for ensuring that a CVD risk evaluation is performed, including, arguably, rheumatologists who are the primary contact point in the healthcare system for patients with systemic rheumatic diseases.

The study by Barber, et al highlights perhaps the most important first step in successful management of high-risk CVD, which is to improve CVD risk factor recording, something that was highly variable in their study ${ }^{4}$. It is reasonable to assume that the risk factor assessment in other clinics is no better and in many cases, likely less frequent than even in this study. The evaluation of CVD risk in these patients is further complicated because, as mentioned above, CVD risk calculators have been developed for the general population but do not accurately predict the risk of future events in patients with $\mathrm{RA}^{4,13}$.

There is also poor awareness in the general medicine community of CVD risk in RA, and there is further lack of formal recognition of the increased risk of CVD in these patients by relevant CV societies, including the European Atherosclerosis Society, European Society of Cardiology, and American Heart Association guidelines, none of which include systemic inflammatory disease as a risk factor contributing to CVD within their scoring system.

Assessment of CVD risk is the first step. Implementation of management recommendations is the vital subsequent step. In addition to management of the underlying systemic inflammation of RA, including efforts to reduce glucocorticoid use and doses, and risk modification, including smoking cessation, weight control, and medical management of HTN and hyperlipidemia, there is a standard global recommendation for physical activity 2 to 3 times per week, reaching $60 \%$ to $70 \%$ of the cardiorespiratory fitness target ${ }^{14,15,16}$. However, the physical limitations that patients may have because of their joint involvement must be considered ${ }^{16}$. Nevertheless, there is a considerable body of evidence that has demonstrated the safety and feasibility of aerobic and resistant exercise for patients with $\mathrm{RA}^{17}$.

To address the shortcomings in assessment of $\mathrm{CV}$ risk, a very few clinics in North America and Northern Europe have established a so-called "cardiorheumatology clinic" for the specific and directed assessment of $\mathrm{CV}$ risk in patients with RA, coupled with integrated risk reduction and management programs ${ }^{18}$. While such efforts hold promise, they may not be practical or affordable in many healthcare settings; nevertheless, they highlight the importance of disease co-management between primary care and rheumatology providers. Future management initiatives will require studies generating data on CVD prevention with lipid treatments and management of the underlying systemic rheumatologic disease for primary and secondary prevention, and in particular to understand whether these measures and treatment goals recommended for the general population are optimal for patients with RA and other rheumatic diseases.

Clearly, better disease assessment, development of valid specific risk algorithms, implementation of guideline recommendations for $\mathrm{CV}$ prevention, and training of specialized personnel - as are implemented for diabetes management - may improve the morbidity and mortality experience of patients with RA and CVD.

ERIC L. MATTESON, MD, MPH,

Division of Rheumatology, Department of Internal Medicine, and Division of Epidemiology, Department of Health Sciences Research Mayo Clinic College of Medicine;

REKHA MANKAD, MD,

Division of Cardiovascular Diseases, Department of Internal Medicine, Mayo Clinic College of Medicine, Rochester, Minnesota, USA.

Address correspondence to Dr. E.L. Matteson, Mayo Clinic and Mayo Foundation, 200 1st St., South West, Rochester, Minnesota 55905, USA; E-mail: matteson.eric@mayo.edu

Personal non-commercial use only. The Journal of Rheumatology Copyright @ 2016 . All rights reserved. 


\section{REFERENCES}

1. Kremers HM, Crowson CS, Therneau TM, Roger VL, Gabriel SE. High ten-year risk of cardiovascular disease in newly diagnosed rheumatoid arthritis patients: a population-based cohort study. Arthritis Rheum 2008;58:2268-74.

2. Wallberg-Jonsson S, Ohman ML, Dahlqvist SR. Cardiovascular morbidity and mortality in patients with seropositive rheumatoid arthritis in Northern Sweden. J Rheumatol 1997;24:445-51.

3. Solomon DH, Curhan GC, Rimm EB, Cannuscio CC, Karlson EW. Cardiovascular risk factors in women with and without rheumatoid arthritis. Arthritis Rheum 2004;50:3444-9.

4. Barber CE, Esdaile JM, Martin LO, Faris P, Barnabe C, Guo S, et al. Gaps in addressing cardiovascular risk in rheumatoid arthritis: assessing performance using cardiovascular quality indicators. J Rheumatol 2016;43:1965-73.

5. Perk J, De Backer G, Gohlke H, Graham I, Reiner Z, Verschuren WM, et al. European guidelines on cardiovascular disease prevention in clinical practice (version 2012): The Fifth Joint Task Force of the European Society of Cardiology and Other Societies on Cardiovascular Disease Prevention in Clinical Practice (constituted by representatives of nine societies and by invited experts). Atherosclerosis 2012;223:1-68

6. Nurmohamed M, EULAR Task Force. EULAR recommendations for cardiovascular risk management in patients with rheumatoid arthritis and other inflammatory joint diseases. EULAR recommendation update on cardiovascular disease in RA. Ann Rheum Dis 2015;74:9.

7. Mancia G, Gagard R, Narkiewicz K, Redón J, Zanchetti A, Böhm M, et al. $2013 \mathrm{ESH} / \mathrm{ESC}$ Guidelines for the management of arterial hypertension: the Task Force for the management of arterial hypertension of the European Society of Hypertension (ESH) and of the European Society of Cardiology (ESC). J Hypertens 2013;31:1281-357.

8. Catapano AL, Chapman J, Wiklund O, Taskinen MR. The new joint EAS/ESC guidelines for the management of dyslipidaemias. Atherosclerosis 2011;217:1.
9. Balgent C, Blackwell L, Emberson J, Holland LE, Reith C, Bhala N, et al. Efficacy and safety of more intensive lowering of LDL cholesterol: a meta-analysis of data from 170,000 participants in 26 randomised trials. Lancet 2010;376:1670-81.

10. Alsheik-Ali AA, Trikalinos TA, Kent DM, Karas RH. Statins, low-density lipoprotein cholesterol, and risk of cancer. J Am Coll Cardiol 2008;52:1141-7.

11. Akkara Vetil BM, Myasoedoca E, Matteson EL, Gabriel SE, Crowson CS. Use of lipid-lowering agents in rheumatoid arthritis: a population-based cohort study. J Rheumatol 2013;40:1082-8.

12. Avina-Zubieta JA, Choi HK, Sadtsafavi M, Etminan M, Esdaile JM, Lacaille D. Risk of cardiovascular mortality in patients with rheumatoid arthritis: a meta-analysis of observational studies. Arthritis Rheum 2008;59:1690-7.

13. Crowson CS, Matteson EL, Roger VL, Therneau TM, Gabriel SE. Usefulness of risk scores to estimate the risk of cardiovascular disease in patients with rheumatoid arthritis. Am J Cardiol 2012;110:420-4.

14. Whitworth JA. Adrenocorticotrophin and steroid-induced hypertension in humans. Kidney Int 1992;37:S34-7.

15. Snowdon $\mathrm{S}$, Nelson $\mathrm{R}$. The effects of nonsteroidal anti-inflammatory drugs on blood pressure in hypertensive patients. Cardio Rev 2011;19:184-91.

16. Semb AG, Kvein TK, DeMicco DA, Fayyad R, Wun CC, LaRosa $\mathrm{JC}$, et al. Effect of intensive lipid-lowering therapy on cardiovascular outcome in patients with and those without inflammatory joint disease. Arthritis Rheum 2012;64:2836-46.

17. de Jong Z, Munneke M, Zwinderman AH, Kroon HM, Jansen A, Ronday $\mathrm{KH}$, et al. Is a long-term high-intensity exercise program effective and safe in patients with rheumatoid arthritis? Results of a randomized controlled trial. Arthritis Rheum 2003;48:2415-24.

18. Rollefstad S, Kvein TK, Holm I, Eirheim AS, Pedersen TR, Semb AG. Treatment to lipid targets in patients with inflammatory joint diseases in a preventive cardio-rheum clinic. Ann Rheum Dis 2013;72:1968-74.

J Rheumatol 2016;43:1947-9; doi:10.3899/jrheum.161025 